\title{
CEFALÉIA INDUZIDA PELA TADALAFILA - RELATO DE CASO E REVISÃO DA LITERATURA
}

\section{TADALAFIL INDUCED HEADACHE - CASE REPORT AND LITERATURE REVIEW}

DOI: $10.5380 /$ rmu.v1i3.40810

Elcio Juliato Piovesan ${ }^{1}$

\section{RESUMO}

Cefaléia secundária ao uso de medicamentos é freqüente e subdiagnosticada na prática médica. Relatamos o caso de um paciente que após o uso de tadalafila desenvolveu uma cefaléia relacionada com atividade sexual. Discutimos os aspectos semiológicos das cefaléias relacionadas com o uso de drogas, com atividade sexual e finalmente comentamos aspectos relacionados com a cefaléia thunderclap primárias e secundárias.

Palavras-chave: Cefaléia Thunderclap; Cefaléia Induzida por Drogas; Cefaléia Relacionada com Atividade Sexual; Tadalafila.

\section{ABSTRACT}

Headache secondary to drug use are frequent and underdiagnosed in daily clinical practice. We report the case of a patient that developed headache related to sexual activity after the use of tadalafil. We discuss the semiologic aspects of the drug induced headache, headaches related with sexual activity, and finally the primary and secondary thunderclap headaches.

Key Words: Thunderclap Headache; Drug Induced Headache; Headache Related with Sexual Activity; Tadalafil. 


\section{INTRODUÇÃO}

Cefaléia é uma das desordens médicas mais prevalentes no consultório médico ${ }^{1}$. 0 adequado manejo destes pacientes é decisivo para melhorar o prognóstico e reduzir impactos sociais e econômicos. A estratégia diagnóstica inclina-se na determinação se estamos frente a uma cefaléia primária ou secundária. A separação entre casos de cefaléia primária das secundárias é fundamental, pois alivia sofrimentos, reduz investigações desnecessárias e otimiza o tratamento de uma maneira mais segura, eficaz e econômica.
Utilizando características semiológicas é possível diferenciar as cefaléias em mais de $95 \%$ dos casos. As principais diferenças entre cefaléias primárias e secundárias foram descritas na tabela 1 . Dentre as cefaléias secundárias, um grupo destaca-se por ser induzida por uso de determinadas substâncias: uso terapêutico (medicamentos); recreativo (drogas ilícitas) ou de forma iatrogênica (intoxicações por drogas ou agentes não ilícitos) ${ }^{2}$.

As cefaléias induzidas por substâncias de uso terapêutico (medicamentos) podem aparecer após uso episódico ou crônico ${ }^{2}$. Na forma episódica o efeito colateral é imediato, aparecendo minutos a horas após

Tabela 1- Características das cefaléias primárias e secundárias

\begin{tabular}{|c|c|c|}
\hline Características & Cefaléia Primária & Cefaléia Secundária \\
\hline Distribuição pela idade & $\begin{array}{l}\text { Inicia na primeira década de vida, } \\
\text { apresenta sua maior prevalência } \\
\text { entre a segunda e quarta décadas e } \\
\text { reduz drasticamente a partir da } \\
\text { quinta década de vida. }\end{array}$ & $\begin{array}{l}\text { Distribuição em qualquer idade, maior } \\
\text { prevalência a partir da quinta década } \\
\text { de vida. }\end{array}$ \\
\hline Recorrência e remissões & $\begin{array}{l}\text { Remissões que variam de dias até } \\
\text { meses }\end{array}$ & $\begin{array}{l}\text { Cefaléias contínuas sem períodos de } \\
\text { remissões. }\end{array}$ \\
\hline Início da dor & $\begin{array}{l}\text { Iniciam leves e progridem } \\
\text { lentamente para maiores } \\
\text { intensidades (esta progressão pode } \\
\text { durar minutos a horas). }\end{array}$ & $\begin{array}{l}\text { Podem aparecer de forma súbita com } \\
\text { padrão Thunderclap (atingem sua } \\
\text { intensidade máxima em menos de } 60 \\
\text { segundos). Este padrão é típico do } \\
\text { aneurisma cerebral roto. }\end{array}$ \\
\hline Evolução da dor & $\begin{array}{l}\text { As cefaléias não progridem durante } \\
\text { o passar do tempo. }\end{array}$ & $\begin{array}{l}\text { Geralmente a dor vai piorando com o } \\
\text { passar dos dias, tornando-se mais } \\
\text { intensa. }\end{array}$ \\
\hline $\begin{array}{l}\text { Horário de início/piora } \\
\text { da cefaléia }\end{array}$ & $\begin{array}{l}\text { Qualquer horário. Uma vez } \\
\text { estabelecida a intensidade máxima, } \\
\text { não oscila durante o dia. }\end{array}$ & $\begin{array}{l}\text { Qualquer horário. Geralmente a dor } \\
\text { piora durante a madrugada. }\end{array}$ \\
\hline Sinais associados & Ausentes & $\begin{array}{l}\text { Febre, perda de peso, claudicação de } \\
\text { mandíbula, perda visual, tinnitus entre } \\
\text { outros }\end{array}$ \\
\hline Exame neurológico & Normal & Geralmente alterado \\
\hline Exames complementares & Normal & Geralmente alterado \\
\hline
\end{tabular}

sua administração. Os pacientes ficam totalmente assintomáticos após o término da ação da droga. $\mathrm{Na}$ forma crônica os pacientes utilizam uma substância terapêutica por muito tempo (diariamente) apresentando uma cefaléia crônica e diária. Nesta forma o paciente não apresenta períodos assintomáticos durante as horas do dia e os dias do mês.

A tadalafila é um potente inibidor do fosfodiesterase do tipo 5 (PDE5), uma enzima encontrada em diversas artérias, sendo que a do corpo cavernoso e do sistema pulmonar destacam-se $\mathrm{e}^{2-3}$. Dentre diversos tipos de efeitos colaterais induzidos pelo seu uso encontra-se a cefaléia. Neste relato de caso descrevemos um paciente que desenvolveu uma cefaléia relacionada com a atividade sexual. Uma revisão dos mecanismos envolvidos e os padrões semiológicos das cefaléias induzidas por drogas serão revisados.

\section{RELATO DE CASO}

Homem com 34 anos, previamente hígido com antecedentes de migrânea com aura de início há 6 anos, com freqüência média de duas a três crises por ano apresenta-se com cefaléia induzida pela tadalafila. 
Aproximadamente 25 minutos após o seu uso e durante a atividade sexual apresenta uma cefaléia que inicialmente caracteriza-se como de leve intensidade, porém adquire uma piora súbita (padrão Thunderclap) atinge o seu pico de intensidade em menos de 60 segundos, com intensidade de 10 pontos na escala verbal analógica da dor. A dor inicia na região occipital e irradia bilateralmente para as regiões temporais e de masseter.

Associado com a dor o paciente não apresentava náuseas, vômitos, fotofobia e/ou fonofobia. Refere uma discreta tontura que melhora após o término da cefaléia. A cefaléia tem uma duração aproximada de 24 horas. As primeiras horas são referidas como de forte intensidade, porém após duas horas a intensidade reduz para leve a moderada. Antecedendo a cefaléia o paciente não referia nenhum tipo de manifestação sensorial ou motor.

O exame físico segmentar e neurológico, incluindo fundo de olho, encontram-se dentro da normalidade. $O$ paciente foi submetido a exame de neuroimagem (Angiorresonância Magnética Cerebral) em fase arterial, venosa e aspectos do parênquima cerebral demonstrando estrutura vascular anômala sem outras alterações (Figura 1).

O paciente foi orientado a utilizar o propranolol como terapia profilática sem aderência. Na revisão de três meses relata mais dois episódios de cefaléia símile aos descritos anteriormente. A interrupção completa da tadalafila foi indicada e o paciente foi acompanhado por mais nove meses não apresentando mais queixas de cefaléia.

O paciente apresentava como investigação suplementar hemograma, glicemia de jejum, função tireoidiana, hepática, renal e metabólitos como sódio, potássio, magnésio todos normais. A utilização da tadalafila era puramente recreativa, uma vez que o paciente não apresentava nenhuma disfunção erétil.

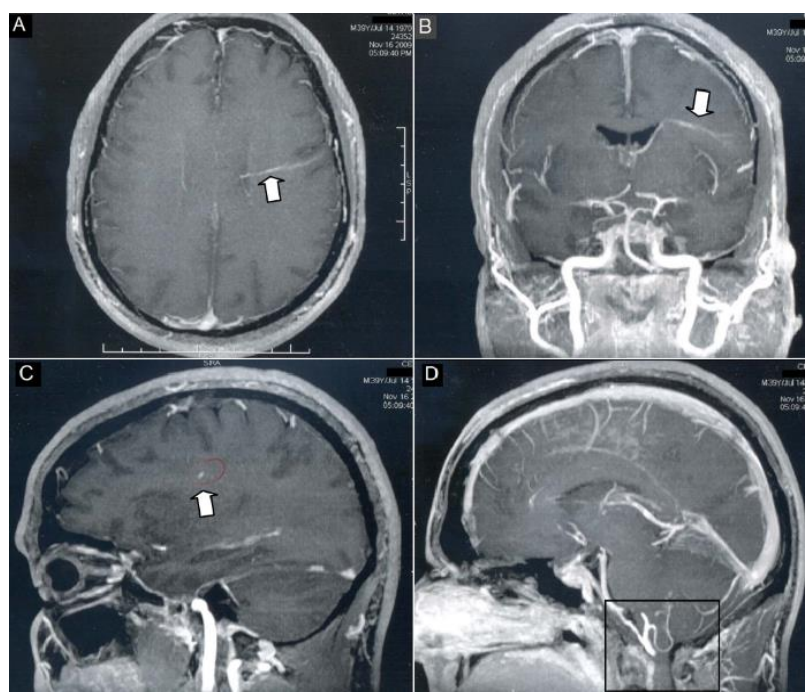

Figura 1: Achados Neuroradiológicos da Ressonância Magnética: A - Corte axial da região frontal do hemisfério cerebral esquerdo com presença de pequena estrutura vascular anômala com sinais de hiperintensidade após injeção de contraste paramagnético; $\mathrm{B}$ - Corte coronal mostrando a veia de drenagem principal (estrutura vascular anômala) projetando-se em direção ao epêndima do ventrículo lateral esquerdo para a veia cerebral interna; C- Corte Sagital mostrando pequena anomalia do desenvolvimento venoso ao nível da região frontal; D Corte sagital mostrando a junção craniocervical dentro da normalidade.

\section{DISCUSSÃO}

O caso descrito alerta o clínico para os riscos da utilização de drogas induzindo cefaléia. Cefaléias crônicas são queixa comuns no convívio dos consultórios e existem inúmeras causas sendo que sempre devemos afastar etiologias farmacológicas dentre elas. A apresentação com um padrão de cefaléia Thunderclap também nos remete ao cuidado neste diagnóstico, pois na sua grande maioria traduz emergências como é o caso dos aneurismas cerebrais.

As cefaléias induzidas por drogas são subdiagnosticadas e ocorrem com uma alta freqüência. O padrão semiológico típico é aquela cefaléia que inicia dentro de minutos a horas após o uso do medicamento e desaparece dentro de até 72 horas após a interrupção do mesmo ${ }^{2}$. O médico deve fazer um inventário farmacológico e sempre estabelecer um nexo causal entre o início de uma determinada droga e o aparecimento da cefaléia. Depois de estabelecido esta relação todos pacientes devem apresentar um exame físico e neurológico normal.

Na literatura médica existe uma variedade de drogas que podem induzir cefaléia (tabela 2). Algumas destas drogas são utilizadas na profilaxia das cefaléias primárias como é o caso da migrânea, outros são utilizadas para outras indicações terapêuticas como hipertensão arterial, artrite reumatóide entre outras ${ }^{4,5}$. As características da cefaléia induzida por drogas são pouco definidas na literatura e evidentemente dependem da droga utilizada. Muitos casos são descritos como uma dor difusa, contínua de moderada a severa intensidade ${ }^{2}$. Não parecem estar relacionadas com náuseas, vômitos, fotofobia e ou fonofobia. A intensidade é variável e depende muito da droga utilizada. 
Tabela 2- Relação de drogas utilizadas por curto ou longo período ${ }^{2-4}$

\begin{tabular}{|ll|}
\hline Acetazolamida & Imunoglobulinas \\
Ácido Nalidíxico & Indometacina \\
Ajmaline & Interferon \\
Amantadina & Isossorbida dinitrato \\
Anti inflamatórios & Isoniazida \\
não esteroidais & \\
Antihistamínicos & Meprobamate \\
Atenolol & Metaqualone \\
Atropina & Metronidazol \\
Barbitúricos & Metoprolol \\
Beta-Interferon & Morfina \\
Bromocriptina & Nifedipina \\
Cafeína & Nimodipina \\
Cálcio-antagonistas & Nitrofurantoína \\
Carbimazol & Nitratos \\
Cinidina & Octreotide \\
Cloroquina & Omeprazol \\
Cimetidina & Ondansetrona \\
Clofibrato & Oxcarbamazepina \\
Codeina & Paroxetina \\
Danazol & Pentoxifilina \\
Didanosina & Perhexilina \\
Digitálicos & Primidona \\
Dihidrolazina & Propranolol \\
Dihidroergotamina & Prostaciclinas \\
Dipiridamole & Ranitidina \\
Disopiramida & Rifampicina \\
Dissulfiram & Sildenafila \\
Estrógenos & Tadalafila \\
Ergotamina & Tamoxifeno \\
Etinilestradiol & Tetraciclinas \\
Gestagens & Teofilina \\
Glicerol trinitratos & Tiamazole \\
Glicosídeos & Trimetropim+Sulfametoxazol \\
Griseofulvin & Triptanos \\
Guanatidina & Verapamil \\
Hidralazina & Vitamina A \\
Imipramina & \\
\hline
\end{tabular}

A cefaléia apresentado pelo nosso paciente foi induzida pelo uso da tadalafila. Todas as características semiológicas de nosso paciente referem-se com uma cefaléia relacionada com a atividade sexual. Entretanto esta relação causal com atividade sexual somente ocorreu após o uso da tadalafila. Desta maneira nosso caso trata-se de uma cefaléia orgástica induzida pela tadalafila.

A cefaléia orgástica ou cefaléia sexual benigna também é conhecida como cefaléia sexual vascular benigna, cefaléia coital ou cefaléia intercurso ${ }^{2}$. Esta cefaleia, como o próprio nome sugere, é precipitada pela atividade sexual. As cefaléias orgásticas possuem um padrão difuso bilateral que se inicia leve e evolui progressivamente, tornando-se subitamente muito intensa (sugerindo ao final desta evolução uma dor do tipo Thunderclap). Cefaléia "Thunderclap" em sua clássica definição são crises que iniciam e atingem seu pico de intensidade em menos de 60 segundos. 0 nosso caso tem um padrão de evolução chamado pseudoThunderclap, pois sua dor inicia-se leve e evolui lentamente, entretanto em um determinado momento sofre um acréscimo súbito (frações de segundo) e muito intenso evoluindo finalmente para uma dor muito severa.

A cefaléia thunderclap pode ser primária (sem etiologia definida) ou secundária a formações aneurismáticas rotas, trombose venosa cerebral, dissecção arterial, síndrome da vasoconstrição cerebral reversível, apoplexia da pituitária, meningites, cisto colóide do terceiro ventrículo, hipotensão liquórica e sinusite aguda (com barotrauma) ${ }^{2}$. A forma thunderclap primária ocorre em outras situações que não relacionadas com causa conhecida como é o caso descrito.

As cefaléias relacionadas com a atividade sexual são subdivididas em dois tipos: pré-orgástica e orgástica. Esta cefaléia ocorre mais prevalentemente no sexo masculino (30: 19) e a idade média destes pacientes é de aproximadamente 49 anos $^{6}$. A cefaléia independe do tipo de atividade sexual e sim com a presença de excitação, podendo ocorrer inclusive durante a masturbação ${ }^{2}$. Durante o curso da cefaléia o paciente pode apresentar manifestações autonômicas. O padrão bilateral ocorre em dois terços dos pacientes, possuindo um padrão difuso ou occipital em $80 \%$ dos $\operatorname{casos}^{2,6}$. A dor é severa ou muito severa em $100 \%$ dos casos, o padrão da dor pode variar entre compressiva, pulsátil, explosiva e muito mais raramente em punhaladas ${ }^{6}$.

A cefaléia relacionada com atividade sexual pode ter uma duração de um minuto até 300 minutos $^{2,6}$. A cefaléia não está relacionada com alterações de consciência, vômitos, tampouco distúrbios visual, motor ou sensorial. Os pacientes com esta cefaléia sempre devem ser investigados com exames de neuroimagem (angiorressonância magnética e/ou angiotomografia cerebral e de vasos cervicais) para descartar causas secundárias ${ }^{2}$.

A tadalafila é um potente inibidor do fosfodiesterase do tipo 5, uma enzima encontrada nos corpos cavernosos promovendo a vasodilatação, utilizado desta maneira para o tratamento da impotência sexual ${ }^{2,3}$. Os efeitos colaterais mais importantes da tadalafila são distúrbios visuais, cefaléia e dor nas costas ${ }^{7}$. Recentemente uma meta-análise envolvendo os oito subtipos dos inibidores do PDE5 disponíveis no mercado farmacêutico mundial demonstrou que o principal efeito colateral é a cefaléia ${ }^{8}$. Dependendo destes estudos a prevalência da cefaléia variou entre 4,6 à $30,4 \%$ dos pacientes utilizando esta classe de medicamento ${ }^{9-14}$. 
Os mecanismos fisiopatológicos desta cefaléia parecem estar diretamente relacionados com o potencial inibidor sobre o PDE5. Estudos recentes mostraram que neurônios dentro do gânglio do trigêmeo expressam o PDE5. Estas células são igualmente relacionadas com o CGRP, um dos importantes neurotransmissores responsáveis pela gênese da dor na migrânea, pois são potentes vasodilatadores e possuem um efeito pronociceptivo (15). O PDE5 atua neuromodulando negativamente e desta maneira produzindo menor efeito neurogênico, protegendo o cérebro destes indivíduos contra a cefaléia primária e secundária.

Desta maneira temos em uma mesma célula (neurônio) o componente pronociceptivo (CGRP) e o componente antinociceptivo (PDE5) interagindo entre si e mantendo um equilíbrio nociceptivo. É racional que quando os inibidores do PDE5 são utilizados a cefaléia ocorra, pois perde-se este efeito antinociceptivo. Em experimentos in-vitro a tadalafila produz uma contração luminal da artéria cerebral média em ratos, e em vivo não produz grandes alterações vasculares intracerebrais ${ }^{3}$. Estes estudos sugerem que a cefaléia induzida pelos inibidores do PDE5 não estão relacionadas com alterações vasculares e sim a alterações neurogênicas dentro dos circuitos nociceptivos trigeminais, o equilíbrio entre pronociceptivo e antinociceptivo ${ }^{16}$.
O efeito antinociceptivo do PDE5 que é bloqueado pelo uso da tadalafila justifica o porquê o paciente apresentava a cefaléia relacionada com atividade sexual exclusivamente quando da utilização deste medicamento.

A presença da malformação venosa encontrada em nosso paciente pode também estar relacionada com a cefaléia, porém ela nunca se manifestou ao longo dos anos neste paciente. A apresentação clínica em nosso caso apareceu exclusivamente quando o paciente utilizou a tadalafila, quando suspendeu o uso desta droga não apresentou mais sintomas álgicos. A cefaléia relacionada com a atividade sexual e alterações venosas têm sido descrita principalmente por alterações do sistema venoso ao nível do seio transverso e do seio jugular. Estas alterações podem promover durante o intercurso da atividade sexual um aumento da pressão intracraniana gerando o quadro álgico ${ }^{6}$. 0 nosso paciente possuía uma alteração venosa que poderia durante o uso da tadalafila potencializar uma pressão intracraniana gerando este quadro de cefaléia.

Em resumo descrevemos o caso de um paciente com cefaléia relacionada com a atividade sexual que foi induzida pelo uso da tadalafila. Novos estudos preclínicos devem ser realizados com o objetivo de avaliar o efeito do PDE5 na neuromodulação nociceptiva.

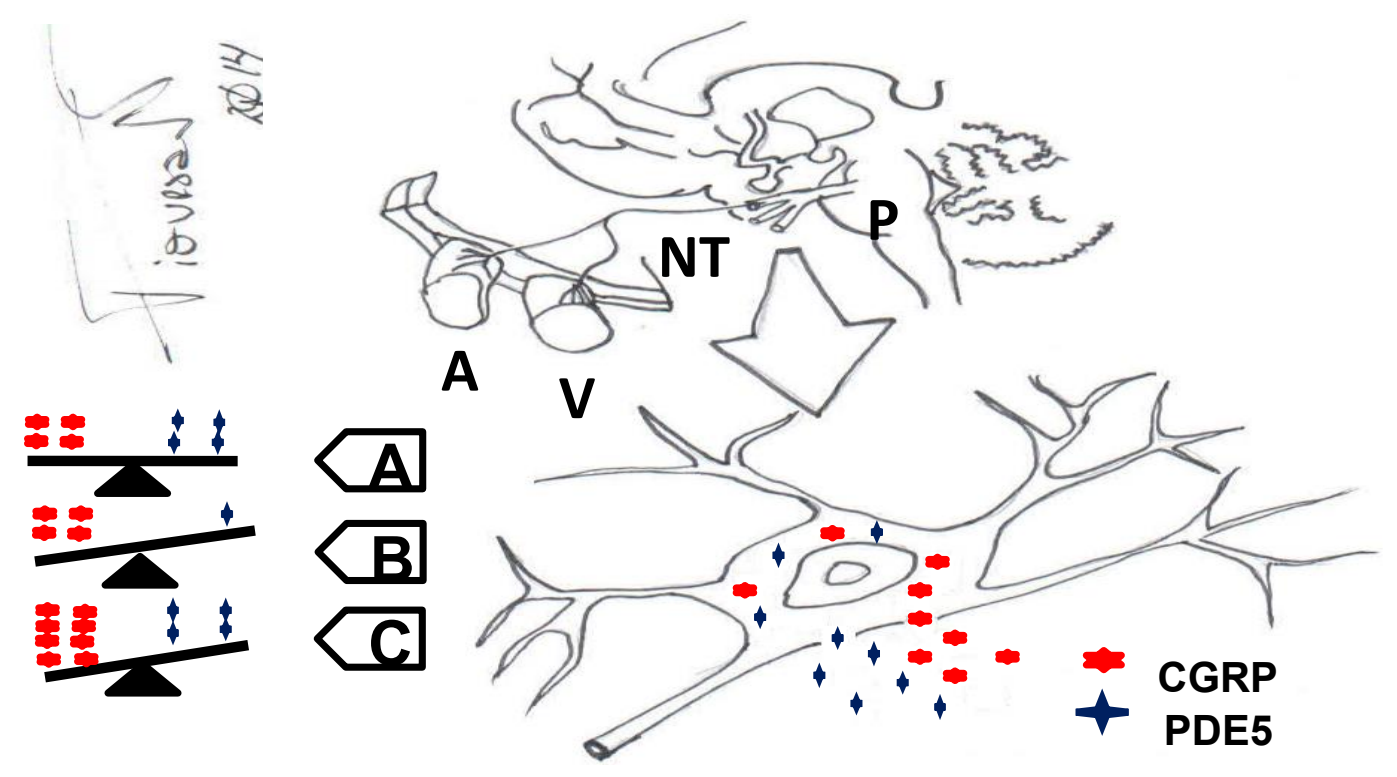

Figura 2: Equilíbrio entre a produção do CGRP e a inibição do PDE5.

Mecanismos no controle nociceptivo cerebral. Situação A - Sem cefaleia: equilíbrio na produção do CGRP e sem inibição do PDE5; Situação B - Bloqueio do PDE5 (Induzido pelo Tadalafila); Situação C - Aumento significativo na produção do CGRP (Comum na migrânea). CGRP - Peptídeo Relacionado com o Gene da Calcitonina; PDE5 - Fosfodiesterase do tipo 5; P - Ponte; NT - Nervo Trigêmeo; A - Artéria meníngea; V - Veia meníngea. A situação B provavelmente ocorreu no paciente relatado. A situação $C$ ocorre normalmente nas crises de migrânea, não tendo nenhuma relação com o PDE5. As alterações sugeridas são produzidas perifericamente nas terminações nervosas e retransmitidas para o tronco cerebral inicialmente para ponte e utilizando com estação intermediária o núcleo caudal do trigêmeo. 


\section{REVISTA MÉDICA DA UFPR}

\section{REFERÊNCIAS BIBLIOGRÁFICAS}

1. World Health Organization. Headache Disorders Fact Sheet. http://www.who.int/mediacentre/factsheets/fs277/en/ Accessed. 8.22.2013

2. The international classification of headache disorders 3rd edition. Headache Classification Committee of the International Headache Society (IHS). Cephalalgia 2013;33:629-808.

3. Krouse C, Gupta S, Nilsson E, Kruse L, Edvinsson L. Differential vasoactive effects of sildenafil and tadalafil on cerebral arteries. Eur J Pharmacol 2012;674:345-351.

4. Askmark H, Lundberg PO, Olsson S. Drug related headache. Headache 1989;29:441-444.

5. Moneiro JM, Dalhof CG. Single use of substances. In: Olesen J, Tfelt-Hansen P, Welch KMA, (eds). The headaches. Lippincott Williams\&Wilkins, Philadelphia 200;861-869.

6. Donnet A, Valade D, Houdart E, et al. Primary cough headache, primary exertional headache and primary headache associated with sexual activity. Neuroradiology 2013;55:297-305.

7. Schultz JE, Dunkem T, Grawlitta-Gorka E, Sorg G. The GAFtandem domain of phosphodiesterase 5 as a potential drug target. Handb Exp Pharmacol 2011;204:151-166.

8. Wang X, Wang X, Liu T, He Q, Wang Y,Zhang X. Systematic review and meta-analysis of the use of phosphodiesterase type 5 inhibitors for treatment of erectile dysfunction following bilateral nerve-sparing radical prostatectomy. PLos One 2014;9:e91327.

9. Brock G, Nehra A, Lipshultz LI, Karlin GS, Gleave M, et. Al. Safety and efficacy of vardenafil for the treatment of men with erectile dysfunction after radical retropubic prostatectomy. J Urol 2003;170:1278-1283.

10. Montorsi F, Nathan HP, McCullough A, Brock GB, Broderick G, et. Al. Tadalafil in the treatment of erectile dysfunction following bilateral nerve sparing radical retropubic prostatectomy: a randomized, double-blind, placebo controlled trial. J Urol 2004;172:1036-1041.

11. Montorsi F, Brock G, Lee J,Shapiro J, Van PH, et. Al. Effect of nightly versus on-demand vardenafil on recovery of erectile function in men following bilateral nerve-sparing radical prostatectomy. Eur Urol 2008;54:924-931.

12. Cavallini G, Modenini F, Vitali G, Koverech A. Acetyl-L-carnitine plus propionyl-L-carnitine improve efficacy of sildenafil in treatment of erectile dysfunction after bilateral nerve-sparing radical retropubic prostatectomy. Urology 2005;66:1080-1085.

13. Padma-nathan H, Eardley I, Kloner RA, Laties AM, Montorsi F. A 4 -year update on the safety of sildenafil citrate (Viagra). Urology 2002;60:67-90..

14. Mulhall JP. The role and structure of a postradical porastatectomy perile rehabilitation program. Curr Urol Rep 2009;10:219-225.

15. Nordgaard JC, Kruse LS, Moller M, Kruuse C. Phosphodiesterases 3 and 5 express activity in the trigeminal ganglion and co-localize with calcitonin gene-related peptide. Cephalalgia 2013;34:503513.
16. Kruuse C, Thomsen LL, Birk S, Olesen J. Migraine can be induced by sildenafil without changes in middle cerebral artery diameter. Brain 2003;126:241-247.

17. Macht, M; Wimbish, T; Clark, BJ; Benson, AB; Burnham, EL; Williams, $A$; et al. Diagnosis and treatment of post-extubation dysphagia: results from a national survey. J Crit Care 2012; 27 (6): 578-86.

18. Splaingard, M; Hutchins, B; Sulton, L; Chaudhuri, G. Aspiration in rehabilitation patients: videofluoroscopic vs bedside clinical assesment. Arch Phys Med Rehabil 1988; 69 (8): 637-640.

19. McGowan, SL; Gleeson, M; Smith, M; Hirsch, N; Shuldham, CM. A pilot study of fibreoptic endoscopic evaluation of swallowing in patients with cuffed tracheostomies in neurological intensive care. Neurocrit Care 2007; 6 (2): 90-93.

20. Cameron, JL; Reynolds, J; Zuidem, GD. Aspiration in patients with tracheotomies. Surg Gynecol Obstet 1973; 136: 68-70.

21. Tolep, K; Getch, CL; Criner, G. Swallowing dysfunction in patients receiving prolonged mechanical ventilation. Chest 1996; 109 (1): 167-172.

22. Reissman, P; Teoh, TA; Cohen, SM, Weiss, EG; Nogueras, JJ; Wexner, SD . Is early oral feeding safe after elective colorectal surgery? A prospective randomized trial. Ann Surg 1995; 222: 73-77.

23. Lassen, K; Revhaug, A. Early oral nutrition after major upper gastrointestinal surgery: why not? Curr Opin Clin Nutr Metab Care 2006; 9: 613-617.

24. Fukuzawa, J; Terashima, H. Early postoperative oral feeding accelerates upper gastrointestinal anastomotic healing in the rat model. World J Surg 2007; 31: 1234-1239.

25. Petrelli, NJ; Cheng, C; Driscoll, D; Rodriguez-Bigas, MA. Early postoperative oral feeding after colectomy: an analysis of factors that may predict failure. Ann Surg Oncol 2001; 8: 796-800.

26. Odderson, R; McKenna, S. A model for management of patients with stroke during the acute phase. Stroke 1993; 24 (12): 1823 1827.

27. American Dietetic Association. Manual of clinical dietetics. 6th ed. Chicago: American Dietetic Association, 2000.

28. Dietitians Association of Australia and The Speech Pathology Association of Australia. Texture-modified foods and thickened fluids as used for individuals with dysphagia: Australian standardised labels and definitions. Nutrition \& Dietetics 2007; 64 (Suppl. 2): S53-S76.

29. Bisch, EM; Logemann, JA; Rademaker, AW; Kahrilas, PJ; Lazarus, CL. Pharyngeal effects of bolus volume, viscosity, and temperature in patients with dysphagia resulting from neurologic impairment and in normal subjects. J Speech Hear Res 1994; 37 (5): 1041-1059.

30. Pardoe, EM. Development of a multistage diet for dysphagia. J Am Diet Assoc 1993; 93 (5): 568-571.

31. Peterson, SJ; Tsai, AA; Scala, CM; Sowa, DC; Sheean, PM; Braunschweig, CL. Adequacy of oral intake in critically ill patients 1 week after extubation. J Am Diet Assoc 2010; 110 (3): 427-33. 